\title{
Application of the Mini-Mental State Examination (MMSE) Scale in Cognitive Assessment of Elderly Patients in a General Ward
}

\author{
Huiling Liu*, Cuiqing Liu, Chuang Hu, Xuling Li, Yahui Zhao, Huan Wang, Chenghuizi Yang \\ Department of Surgery, The First Affiliated Hospital of Jinan University, Guangzhou, China
}

\begin{abstract}
[Abstract] Objective: To analyze the application of the Mini-Mental State Examination (MMSE) scale in cognitive assessment of elderly patients in a general ward. Methods: Elderly patients who underwent nephrosurgery and thoracic surgery between July 2015 to June 2019 were eligible for the study. A total of 713 patients enrolled and data were collected for the assessment of cognitive status. We analyzed the relationship between cognitive impairment, degree of cognitive impairment, age, and gender. Result: In the 713 cases of cognitive ability assessment by the MMSE, there was cognitive impairment in $21(4.04 \%)$ male patients, and 10 (5.18\%) female patients. In addition, the analysis of the correlation between cognitive impairment and gender showed a P value of 0.51 , which was not statistically significant. In the participants age research, the average age of the patients in the cognitive normal group was $72.30 \pm 6.50$ years old, and the average age of the patients in the cognitive impairment group was $78.61 \pm 6.90$ years old. Their $p$ value was $<0.0001$, which was statistically significant. Conclusion: There is a correlation between cognitive impairment and age. Therefore, the MMSE helps detect cognitive impairment and potential safety hazards in elderly patients. It is convenient for medical staff to take corresponding measures in time to ensure the safety of the patients.
\end{abstract}

Keywords: Old people; Cognitive function; Mini-Mental State Examination

Publication date: May, 2021; Publication online: 31 May, 2021

*Corresponding author: Huiling Liu, Liuyxaa2021@163.com

\section{Introduction}

Elderly patients show progressive systemic organ decline due to older age leading to a great impact on their cognitive function ${ }^{[1]}$. Based on the research, reports show that cognitive decline in hospitalized elderly patients amounted to $47.5 \%$ [2]. According to the result of previous epidemiologic surveys, the prevalence of cognitive impairment in elderly patients aged 65 years was quite high and ranged from $35 \%$ to $50 \%$ of the population ${ }^{[3]}$. This could lead to delicate and persistent cognitive impairment in elderly patients after surgery ${ }^{[4]}$. In addition, cognitive impairment is the transitional stage from the cognitive changes of normal aging to very early dementia ${ }^{[5]}$. The research shows that $10-15 \%$ of patients with mild cognitive impairment (MCI) progress to dementia annually compared to $1-2 \%$ of healthy controls ${ }^{[6]}$.

Originally, the Mini-Mental State Examination (MMSE) was designed to screen for dementia of Alzheimer's [7]. Currently, it is used to evaluate various cognitive impairments in medical organization, and can detect mild cognitive impairment with higher sensitivity. This assessment not only has high test-retest reliability and good internal consistency but also has strength in detecting executive function ${ }^{[8,9]}$. The MMSE includes a 30-point scale which is used in various languages. The researchers need spend about 5 to 10 mins to finish the test. For cut-off scores for dementia, the patients are commonly considered to have dementia if their scores were 24 to 26 out of $30^{[10,11]}$. The aim of this study was to evaluate the application of the 
MMSE scale in cognitive assessment of elderly patients in a general ward.

\section{Methods}

\subsection{Participants Enrollment and Survey Methods}

We invited 713 elderly patients who were treated in department of renal surgery and thoracic surgery between July 2015 to June 2019 and were older than 65 years. The patients were divided into a cognitively normal group $(\mathrm{n}=$ 682 ) and cognitively impaired group $(n=31)$, according to their cognitive function. All participants finished the MMSE after the patient file was established face to face.

The MMSE included six aspect tests based on directivity, immediate memory, attention and computation, delayed memory, language, and visual space. There were 30 questions in the scale. Each correct answer was equivalent to 1 point. Each incorrect or no answer was equivalent to 0 points. The scoring was as follows: scores of 27-30 were considered as normal cognition; 21-26 was classified as mild cognitive impairment; $10-20$ is classified as moderate cognitive impairment; and 0-9 were classified as severe cognitive impairment ${ }^{[3]}$. The retest reliability was $0.80-0.99$, the sensitivity of screening was $80 \%-90 \%$, and the specificity was $70 \%-80 \%{ }^{[4]}$.

\subsection{Statistical Analysis}

In this study, Microsoft Office Excel 2010 software was used to build a database, and double entry was made for the final recovered data. The software performed logical verification to ensure the accuracy of regression entry. In addition, SPSS 22.0 software was used for data analysis and processing. Statistical analysis methods included a univariate analysis - the categorical variables were assigned by the value method and tested by a chi-square test, and continuous variables were tested by an independent sample t-test. Single factor analysis of variance (ANOVA) was used to compare the means of multiple groups. Bilateral $\alpha=0.05$ was taken as the test level, and $\mathrm{P} \leq 0.05$ was considered as statistically significant.

\section{Results}

A total of 713 patients were included in this study which included 520 males and 193 females. There were 31 patients with cognitive impairment and 682 patients with normal cognition (Table 1). In addition, among the patients with cognitive impairment, there were $21(4.04 \%)$ male patients and $10(5.18 \%)$ female patients. Statistical analysis showed no significant difference between cognitive impairment and gender differences.

Table 1. Correlation between cognitive impairment and gender

\begin{tabular}{ccccc}
\hline Projects & Male & Female & Total & P value \\
\hline cognitively normal group & 499 & 183 & 682 & 0.51 \\
cognitively impaired group & 21 & 10 & 31 & 713 \\
Total & 520 & 193 & & \\
\hline
\end{tabular}

Table 2 shows the effect of age on cognitive impairment. The results indicated that the average age of the patients was $72.30 \pm 6.50$ years old in the cognitive normal group, and $78.61 \pm 6.90$ years old in the cognitive impairment group. We compared the mean age between the two groups via an independent sample $\mathrm{T}$ test. The results showed that the difference between the two groups was statistically significant.

Table 2. Correlation between cognitive impairment and age

\begin{tabular}{ccccc}
\hline Projects & Age $($ year $)($ Mean \pm SD) & SD & N (case) & P value \\
\hline cognitively normal group & $72.30 \pm 6.50$ & 6.496 & 682 & $<0.0001 \#$ \\
cognitively impaired group & $78.61 \pm 6.90$ & 6.903 & 31 & \\
\hline
\end{tabular}

\# stands for independent sample T test

In order to explore whether there was a gender correlation between patients with different degrees of cognitive impairment and patients with normal cognition, we divided the subjects into different groups of normal 
cognition, mild cognitive impairment, moderate cognitive impairment, and severe cognitive impairment. We found that the proportion of male patients in the four groups were $96.96 \%$ (499/520), $1.54 \%(8 / 520), 1.15 \%$ (6/520), $1.35 \%$ (7/520); while female patients in the four groups were $94.82 \%$ (183/193), $1.04 \%$ (2/193), $1.55 \%$ (3/193), $2.59 \%$
(5/193) (Table 3). There were no significant gender differences between the mild, moderate, and severe cognitive impairment groups and the normal cognitive impairment group. Furthermore, different degrees of cognitive impairment were not associated with normal cognitive impairment and gender.

Table 3. Correlation between degree of cognitive impairment and Gender

\begin{tabular}{ccccccc}
\hline \multirow{2}{*}{ Gender } & \multicolumn{3}{c}{ Cognitive grade grouping } & N (case) & P value \\
\cline { 2 - 7 } & $\begin{array}{c}\text { Severe cognitive } \\
\text { impairment }\end{array}$ & $\begin{array}{c}\text { Moderate } \\
\text { cognitive } \\
\text { impairment }\end{array}$ & $\begin{array}{c}\text { Mild cognitive } \\
\text { impairment }\end{array}$ & $\begin{array}{c}\text { Cognitively } \\
\text { normal }\end{array}$ & & \\
\hline Male & 7 & 6 & 8 & 499 & 520 & $0.63^{*}$ \\
Female & 5 & 3 & 2 & 183 & 193 & 713 \\
Total & 12 & 9 & 10 & 682 & \\
\hline
\end{tabular}

* stands for chi-square test

Based on the results of correlation between degree of cognitive impairment and age, the mean age of the four groups was $72.30 \pm 6.50$ years, $79.00 \pm 5.908$ years, $79.56 \pm 9.084$ years, and $77.30 \pm 9.084$ years, respectively. Additionally, there were statistically significant differences in age between the cognitive-normal group and the cognitive-impairment group at different levels, such that the average age of the three groups was higher than that of the cognitive-normal group. Therefore, patients with any degree of cognitive impairment were older than those with normal cognitive impairment (Table 4).

Table 4. Correlation between degree of cognitive impairment and age

\begin{tabular}{cccc}
\hline Projects & Age (year) (Mean \pm SD) & N (case) & P value \\
\hline Cognitively normal & $72.30 \pm 6.50$ & 682 & $<0.01 *$ \\
Mild cognitive impairment & $79.00 \pm 5.908$ & 12 & $<0.01 *$ \\
Moderate cognitive & $79.56 \pm 9.084$ & 9 & $<0.01 *$ \\
impairment & $77.30 \pm 9.084$ & 10 & \\
Severe cognitive impairment & & 713 & \\
Total & & & \\
\hline
\end{tabular}

* represents the comparison of mean values between groups, using one-way analysis of variance (one-way ANOVA)

\section{Discussion}

As the global aging population increases, the number of elderly people in China will reach 254 million by the end of $2019^{[12]}$. Based on the research, in China, the number of elderly people with cognitive impairment is also increasing due to aging, leading to an increase in the annual number of hospitalized elderly patients ${ }^{[3]}$. The number of elderly patients in general wards are also increasing significantly ${ }^{[13]}$. In fact, elderly patients with cognitive impairment face multiple safety hazards when they are in the hospital, leading to many difficulties in treatment and recovery, such as longer hospital stays and higher medical costs ${ }^{[14]}$. Therefore, timely detection and identification of disorders in elderly patients and the adoption of appropriate measures can avoid the occurrence of accidents during hospitalization of elderly patients. Shortening the length of hospital stay can reduce the medical costs, which is of positive significance to the patients, their families, the hospital, and society.

Based on the above results, there was no correlation between gender and cognitive ability in the elderly patients 
in the general ward. Therefore, both male and female elderly patients needed to use the MMSE for cognitive ability assessment. In addition, the cognitive abilities of older patients are age-related. Results showed that the average age of the elderly patients in the cognitive impairment group was significantly higher than that in the normal cognitive group, which means the risk of cognitive impairment in elderly patients increases with age. In fact, there were more subtle differences in cognitive impairment among patients of the same age; hence, investigators need better assessment techniques to accurately identify patients with different levels of cognitive impairment and to provide more accurate data.

Furthermore, elderly patients have varying degrees of cognitive impairment. However, most family members of elderly patients did not realize this risk and paid little attention to the safety hazards of elderly patients in their daily lives. The medical staff should inform the family immediately when elderly patients are diagnosed with cognitive impairment, and the staff should explain to the patient's family the potential safety problems and corresponding countermeasures and sign the safety notice. In addition, the medical staff should confirm the bedside label and the patient's wrist band label to ensure the safety of elderly patients.

\section{Conclusion}

There is a correlation between cognitive impairment and age. Therefore, the MMSE helps to detect cognitive impairment and potential safety hazards in elderly patients. It is convenient for medical staff to take corresponding measures in time to ensure the safety of patients.

\section{References}

[1] John A, Patel U, Rusted J, et al., 2019, Affective Problems and Decline in Cognitive State in Older Adults: A Systematic Review and Meta-Analysis. Psychol. Med., 49(3): 353-365.

[2] Saez De Asteasu ML, Martinez-Velilla N, Zambom-Ferraresi F, et al., 2020, Cognitive Function Improvements Mediate Exercise Intervention Effects on Physical Performance in Acutely Hospitalized Older Adults. Journal of the American Medical Directors
Association.

[3] Zhou Z, Mao F, Han Y, et al., 2020, Social Engagement and Cognitive Impairment in Older Chinese Adults: The Mediating Role of Psychological Well-Being. Journal of Aging and Health, 32(7-8): 573-581.

[4] Charipova K, Urits I, Viswanath O, et al., 2020, Preoperative Assessment and Optimization of Cognitive Dysfunction and Frailty in the Ambulatory Surgical Patient. Current Opinion in Anesthesiology, 33(6): 732-739.

[5] Racine AM, Fong TG, Gou Y, et al., 2018, Clinical Outcomes in Older Surgical Patients with Mild Cognitive Impairment. Alzheimers \& Dementia, 14(5): 590-600.

[6] Ciesielska N, Sokolowski R, Mazur E, et al., 2016, Is the Montreal Cognitive Assessment (MoCA) Test Better Suited than the Mini-Mental State Examination (MMSE) in Mild Cognitive Impairment (MCI) Detection Among People Aged Over 60? Meta-analysis. Psychiatr. Pol., 50(5): 1039-1052.

[7] Arevalo-Rodriguez I, Smailagic N, Figuls MRI, et al., 2015, Mini-Mental State Examination (MMSE) for the Detection of Alzheimer's Disease and Other Dementias in People with Mild Cognitive Impairment (MCI). Cochrane Database Syst Rev., (3): 76.

[8] Bejanin A, Schonhaut DR, La Joie R, et al., 2017, Tau Pathology and Neurodegeneration Contribute to Cognitive Impairment in Alzheimer's Disease. Brain, 140: 3286-3300.

[9] Montenigro PH, Alosco ML, Martin BM, et al., 2017, Cumulative Head Impact Exposure Predicts Later-Life Depression, Apathy, Executive Dysfunction, and Cognitive Impairment in Former High School and College Football Players. J. Neurotrauma, 34(2): 328-+.

[10] Chapko D, Mccormack R, Black C, et al., 2018, Life-Course Determinants of Cognitive Reserve (CR) in Cognitive Aging and Dementia - A Systematic Literature Review. Aging Ment. Health, 22(8): 915-926.

[11] Hershkovitz A, Kornyukov N, Brill S, 2017, Place Orientation and Visual Construction Subdomains of The Mini Mental State Examination Test As Predictors of Rehabilitation Outcome of Post-Acute Hip-Fractured Patients. Disability and Rehabilitation, 39(22): 2339-2345.

[12] Zhang J, Xu X, Yang L, et al., 2021, Met and Unmet 
Care Needs of Home-Living People with Dementia in China: An Observational Study using the Camberwell Assessment of Need for the Elderly. Geriatrics \& Gerontology International, 21(1): 102-107.

[13] Nayeri ND, Gholizadeh L, Mohammadi E, et al., 2015, Family Involvement in the Care of Hospitalized Elderly Patients. Journal of Applied Gerontology, 34(6):
779-796.

[14] Gan S, Yu Y, Wu J, et al., 2020, Preoperative Assessment of Cognitive Function and Risk Assessment of Cognitive Impairment in Elderly Patients with Orthopedics: A Cross-Sectional Study. BMC Anesthesiology, 20(1). 